\title{
The Evolutionary Evidence for Be/Black Hole Binaries
}

\author{
Natalya V. Raguzova and Vladimir M. Lipunov \\ Sternberg Astronomical Institute, Moscow, 119899 Russia \\ Faculty of Physics, Moscow University, Moscow, 117234 Russia
}

\begin{abstract}
Using a Monte Carlo simulation of the modern scenario of the evolution of binary systems (the "Scenario Machine"), we calculate the number of binary black holes with Be stars and their expected observational properties. So far, only two possible candidates for $\mathrm{Be} / \mathrm{BH}$ binaries have been proposed among the observable sources, the superluminal source GRS $1915+105$ in the Galaxy and RX J0117.6-7330 in the SMC. We obtained an evolutionary track that can lead to the formation of such systems. The modern evolutionary scenario predicts the existence of binary black holes on eccentric orbits around Be stars and such systems may be discovered in the near future.
\end{abstract}

\section{Introduction}

A discovery of binary black holes with massive Be stars would be of great importance for the modern theory of stellar evolution. Only a handful of reliable candidates for binary black holes with optical companions are known at present. They must all be products of the evolution of binary systems with massive companions. So far, two "currently active" possible candidates for $\mathrm{Be} / \mathrm{BH}$ binaries have been proposed among the observable sources, the source GRS 1915+105 in the Galaxy (Mirabel et al. 1997) and RX J0117.6-7330 in the SMC (Clark et al. 1997). In this paper we calculate the expected distributions of $\mathrm{Be} / \mathrm{BH}$ systems over orbital periods and eccentricities for different scenario parameters using a Monte Carlo simulation of the binary evolution and discuss possible ways in which such systems can be formed.

\section{Be+BH versus $B e+N S$ " $A$ " in our Galaxy}

The important point is that the collapse of a massive star into a black hole (BH) can be asymmetrical, so that the newborn $\mathrm{BH}$ can acquire an additional, presumably randomly oriented in space, kick velocity $w$. In view of the importance of this parameter, we study the influence of this asymmetry on the evolutionary calculations for the Maxwellian and Lyne \& Lorimer (1994) kick distributions. The observed number of Be/NS "A" systems in the Galaxy is equal to about 25 (we include transient Be/X-ray binaries such as A0535+26, X Per, 4U1145-619 etc.). Thus, the expected "observed number" of Be/BH transients in the Galaxy is equal to unity only for certain parameters of black hole formation. 
Given our limited current understanding of the black hole formation processes, we allow a fraction of the presupernova mass $\left(M_{*}\right)$ collapsing to a $\mathrm{BH}$, $k_{B H}=M_{B H} / M_{*}=0.5$. As a separate independent criterion we included the calculated Galactic number of black hole candidates with evolved OB-companions. We adopt a lower limit to the initial mass of the star collapsing to a black hole, $M_{c r}$, to vary in the range from 50 to $80 \mathrm{M}_{\odot}$.

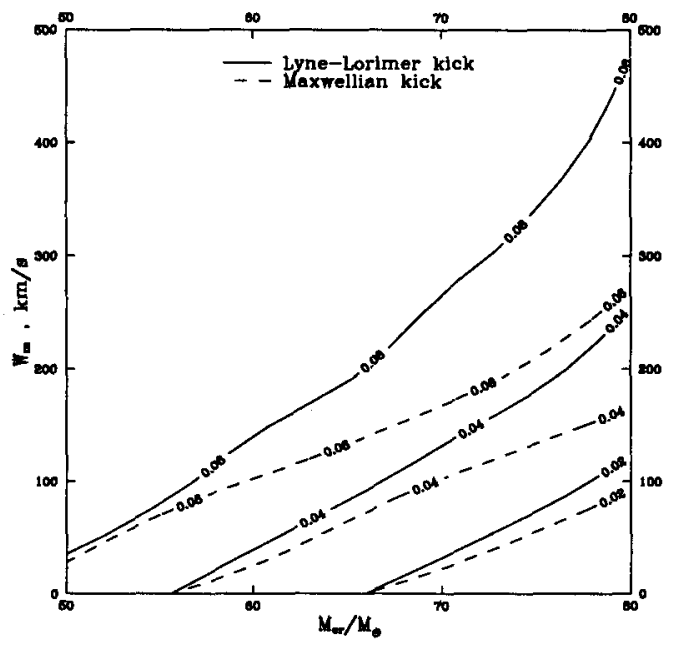

Figure 1. The "Curves of the best correspondence" for the scenario parameters (see description in the text)

The results of our calculations in the form of the "curves of best correspondence" are presented in Figure 1. These represent the set of possible values of scenario parameters of binary systems. For these parameters the calculated ratio of the number of $\mathrm{Be} / \mathrm{BH}$ to $\mathrm{Be} / \mathrm{NS}$ " $\mathrm{A}$ " systems agrees with the accepted observational value of $1 / 25$. Taking into account that this value is only known to within a factor of 2 , we also plot in Figure 1 the curves corresponding to the ratios $\mathrm{Be} / \mathrm{BH}$ and $\mathrm{Be} / \mathrm{NS}$ " $\mathrm{A}$ " of $2 / 25$ and $1 / 50$, respectively.

\section{The expected distributions of $\mathrm{Be} / \mathrm{BH}$ binaries over orbital param- eters}

Based on the restrictions to the scenario parameters shown in Figure 1 we have calculated the expected distributions of the number of $\mathrm{Be} / \mathrm{BH}$ systems over eccentricities and orbital periods. These parameters have been chosen as corresponding with the "curves of best correspondence", that is, we adopt the observed number of $\mathrm{Be} / \mathrm{BH}$ binaries in our Galaxy to be unity. The distributions we obtain are shown in Figure 2. In Figure 2 we can see that the expected orbital periods of $\mathrm{Be} / \mathrm{BH}$ binaries lie in the range from several days to several years with a maximum at several tens of days. The expected eccentricities of 

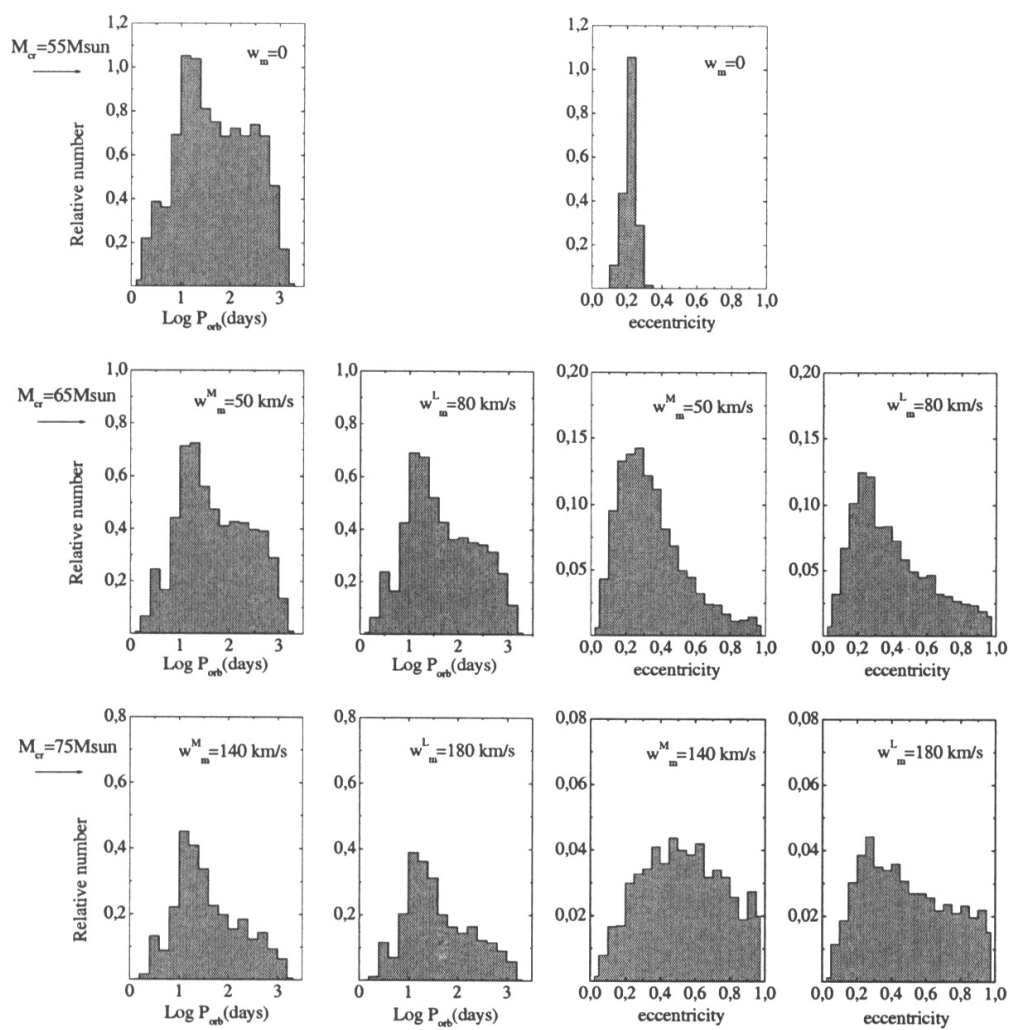

Figure 2. Expected distribution of number of $\mathrm{Be} / \mathrm{BH}$ binaries over orbital parameters

such systems fall in a broad range of values and depend strongly on the value of kick velocity. With increasing additional kick velocity the number of binaries with high values of eccentricity increases and the maximum of the distribution shifts to large eccentricities.

\section{Evolutionary scenario for high-eccentricity $\mathrm{Be} / \mathrm{BH}$ binaries}

Here we present an example of the "Scenario Machine" (Lipunov et al. 1996a) computations for the formation of a Be star paired with a black hole. The evolutionary track that can lead to the formation of a $\mathrm{Be}+\mathrm{BH}$ binary is presented in Figure 3. Here we use the following notation for the evolutionary stages of the components: $\mathrm{MS}=\mathrm{a}$ main sequence star inside its Roche lobe; a MS-star that accretes matter during the first mass transfer is considered to be a rapidly rotating Be-star if its mass is more than $10 \mathrm{M}_{\odot}$; Giant = an evolved giant not filling its Roche lobe; WR = a helium (Wolf-Rayet) star; $\mathrm{RLO}(\mathrm{f})=$ Roche lobe 


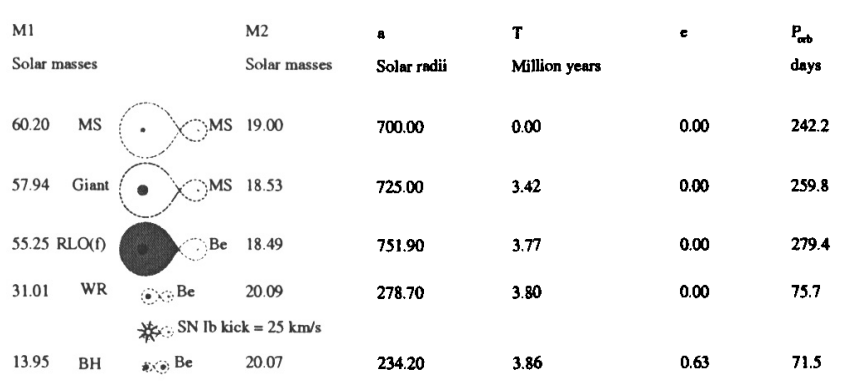

Figure 3. Possible evolutionary track leading to $\mathrm{Be} / \mathrm{BH}$ binary formation

overflow on a thermal timescale; $\mathrm{BH}=$ black hole; $\mathrm{SN} \mathrm{Ib}=$ core collapse of a star with initial mass $M>10 \mathrm{M}_{\odot}$ in a binary with $a<200 \mathrm{AU}$. When calculating evolutionary tracks we assumed anisotropic collapse with $w_{m}=25 \mathrm{~km} \mathrm{~s}^{-1}$. The age of the system according to our evolutionary scenario is $4 \times 10^{6} \mathrm{yr}$.

\section{Conclusion}

In the framework of different assumptions about the parameters of binary evolution scenario we have estimated the relative number of binary black holes with Be star companions, which is not subject to observational selection effects. For probable parameters of black hole formation we obtained the expected number of $\mathrm{BH}+\mathrm{Be}$ binaries to be of order of unity per 20-30 Be/NS "A" systems. The values of obtained kicks satisfy previous calculations of the binary radiopulsar population based on more general observational data (Lipunov et al. 1996b). This means that such binary black holes may be discovered in the near future. All such systems must be highly eccentric, with orbital periods lying in a range of 10 days to several years.

Acknowledgments. This work is partially supported by the RFBR grant 98-02-16801, by the NTP program "Astronomija" (project 1.4.2.3) of Ministry of Science and High Technology and by the "Universitety Rossii" grant 5559.

\section{References}

Clark, G.W., Remillard, R.A., Woo, J.W. 1997, ApJ 474, L111

Lipunov, V.M., Postnov, K.A., Prokhorov M.E. 1996a, Astrophys. and Space Phys. Reviews 17, 1

Lipunov, V.M., Postnov, K.A., Prokhorov M.E. 1996b, A\&A 310, 489

Lyne, A.G., Lorimer, D.R. 1994, Nature 369, 127

Mirabel, I.F., Bandyopadhyay, R., Charles, P.A., et al. 1997, ApJ 477, L45 